\title{
Assessing cardiorespiratory capacity in older adults with major depression and Alzheimer disease
}

\author{
Avaliação da capacidade cardiorrespiratória em idosos \\ com depressão maior e doença de Alzheimer \\ Marcos Felipe Zanco ${ }^{1,2}$, Helena Moraes², Geraldo Maranhão Neto ${ }^{3}$, Jerson Laks ${ }^{4,5}$, Andrea Camaz Deslandes ${ }^{2,6}$
}

\section{Keywords}

Aging, mental health, physical fitness.

\section{Palavras-chave}

Envelhecimento, saúde mental, aptidão física.

\begin{abstract}
Objective: To assess cardiorespiratory capacity through subjective and objective tests in older adults diagnosed with major depression (MDD), Alzheimer disease (AD) and healthy older adults. Methods: Fifty seven subjects (72 \pm 7.9 years) were divided into three groups: MDD $(n=20), A D(n=17)$ and Healthy $(n=20)$. The subjects answered Hamilton Scale (HAM-D), Mini-Mental State Examination (MMSE), Veterans Specific Activity Questionnaire (VSAQ) and 2-minute Step test. Results: MDD and AD showed lower scores than healthy group for Nomogram VSAQ $(p<0.001)$ and 2-minute Step $(p=0.009 ; p=0.008$, respectively). Adjusted for age and educational level, no differences among groups were observed for Step (MDD, $p=$ 0.097; $A D, p=0.102$ ). $A D$ group did not present differences to healthy group for Step, when adjusting for MMSE $(p=0.261)$. Conclusions: Despite the lower cardiorespiratory fitness of elderly patients with DM and DA have been found in both evaluations, the results should be viewed with caution, since the tests showed low correlation and different risk classifications of functional loss. In addition, age, level educational and cognitive performance are variables that can influence the performance objective evaluation.
\end{abstract}

\section{RESUMO}

Objetivo: Avaliar a capacidade cardiorrespiratória em idosos com depressão maior (DM), doença de Alzheimer (DA) e saudáveis, subjetiva (nomograma VSAQ) e objetivamente (2-minute STEP). Métodos: Cinquenta e sete idosos (72 $\pm 7,9$ anos) foram divididos em três grupos: DM ( $n$ $=20)$, DA $(n=17)$ e saudáveis $(n=20)$. Os sujeitos responderam a escala de Hamilton (HAM-D), Miniexame do Estado Mental (MEEM), VSAQ (Veterans Specific Activity Questionnaire) e o teste 2-minute Step. Resultados: DM e DA apresentaram menores escores comparados aos saudáveis no nomograma VSAQ $(p<0,001)$ e no teste Step $(p=0,009$ e $p=0,008)$. Controlado por idade e escolaridade, não houve diferenças entre os grupos no Step (DM, $p=0,097$; DA, $p=0,102$ ). DA também não mostrou diferenças no STEP em comparação aos saudáveis, quando controlado pelo MEEM $(p=0,261)$. Conclusões: Apesar de a menor aptidão cardiorrespiratória de idosos com DM e DA ter sido encontrada nas duas avaliações, os resultados devem ser observados com cautela, uma vez que os testes apresentaram baixa correlação e classificações de risco de perda funcional divergentes. Além disso, a idade, o nível educacional e a capacidade cognitiva são variáveis que podem influenciar o desempenho na avaliação objetiva.

\footnotetext{
1 Universidade Federal do Rio de Janeiro (UFRJ), Programa de Pós-graduação em Educação Física. 2 Universidade Federal do Rio de Janeiro (UFRJ), Laboratório de Neurociências do Exercício (LaNEx). 3 Universidade Salgado de Oliveira (Universo).

4 Universidade Federal do Rio de Janeiro (UFRJ), Instituto de Psiquiatria (IPUB), Centro de Doença de Alzheimer e outras Desordens Mentais na Velhice (CDA).

5 Conselho Nacional de Desenvolvimento Científico e Tecnológico (CNPq).

6 Universidade do Estado do Rio de Janeiro (Uerj), Instituto de Educação Física e Desportos (IEFD).
} 


\section{INTRODUCTION}

During the aging process, there is an increase in the prevalence and incidence of mental disorders, such as depression and dementia. The prevalence of major depression in the elderly people ranges from $0.9 \%$ to $9.4 \%$ in private households'. A study with 7.040 Brazilian elderly people found a prevalence of $22.7 \%$ of depressive symptoms². Besides depression, the prevalence of neurodegenerative disorders also increases with aging. In this sense, dementias are the most prevalent, ranging from $1.6 \%$ in elderly people aged 65 years to $38.9 \%$ in those aged 85 years and older ${ }^{3}$, with Alzheimer's disease (AD) being the most common. Cognitive impairment and depressive symptoms can influence the risks of physical decline and cardiorespiratory fitness, increasing mobility impairment and further loss of independence $^{4-6}$.

Among the physical skills damaged with aging, cardiorespiratory capacity reduces 9\%-10\% for each decade, contributing to the decrease of functional capacity ${ }^{7}$, which demonstrates the importance of assessing this valence, especially in older adults with physical and brain disorders. The gold standard for assessing maximal aerobic capacity is to directly measure maximal oxygen intake $\left(\mathrm{VO}_{2 \text { max }}\right)$. However, the use of this technique in epidemiological studies is limited, especially due to high cost equipment, the need for technical expertise, risks associated with maximal exercise, especially in older adults, and possible physical limitations ${ }^{8-10}$.

Alternatives for the estimation of exercise capacity have been developed over the years. One of them is the 2-minute Step, an aerobic resistance test included in the battery of tests named Senior Fitness Test ${ }^{11}$. Its objective is to assess physiologic parameters that support physical mobility and, therefore, the functional independence of elderly adults. Another possibility to measure cardiorespiratory fitness is through questionnaires, without exercise testing. Created specifically for elderly subjects, the Veterans Specific Activity Questionnaire (VSAQ) is a questionnaire-based evaluation method developed to easily estimate the maximal aerobic capacity, in metabolic equivalents (METS). Based on this questionnaire, a regression equation reflecting the relationship between achieved METS by VSAQ and age results in a nomogram. This nomogram has allowed more accurate estimates regarding patient exercise capacity ${ }^{12}$, by significantly improving the estimation of both the projected and measured peak $\mathrm{VO}_{2}{ }^{10}$. Studies applying the VSAQ show an acceptable prediction of patient exercise capacity ${ }^{10,13}$, a strong prediction of all-cause mortality in patients referred to exercise testing ${ }^{14}$, and significant correlations between estimated METs and the 6-min walk test, an aerobic resistance test9,15,16.

Despite the fact that previous studies have shown a wellestablished VSAQ application in elderly people, there are no accounts in the literature investigating its use with older in- dividuals diagnosed with major depressive disorder (MDD) or AD. The subjectivity of this measurement may contribute to alterations in score by mood disorders and cognitive status impairment. Furthermore, few studies in the literature are available associating subjective and objective cardiorespiratory capacity assessments, especially involving clinical populations, such as MDD and AD individuals. The purposes of the present study were to: 1) assess the relationship between the nomogram VSAQ and 2-minute Step in older adults diagnosed with either MDD, AD and healthy older adults; 2) analyze the influence of depressive symptoms and cognitive capacity on the differences among the groups for each variable; 3 ) compare the results of the 3 groups for all tested variables. We hypothesized that associations between both variables would be present only in the Healthy group, since mental disorders produce impairment in objective and subjective test performances and, consequently, depressive symptoms and cognitive capacity would be responsible for the possible differences among the groups.

\section{METHODS}

\section{Study design and sample selection}

In this descriptive, observational and cross-sectional study, 57 elderly subjects ( $>60$ years old) were recruited (20, with MDD; 17, with AD; and 20 healthy). Both MDD and AD subjects were conveniently recruited at Center for Alzheimer's Disease and Related Disorders, Psychiatry Institute, Federal University of Rio de Janeiro (CDA-IPUB-UFRJ). Clinical Dementia Rating (CDR) was applied to quantify the severity of dementia symptoms in AD group (mild, CDR $1=70.6 \%$; moderate, CDR $2=29.4 \%$ ). The healthy subjects were recruited at the Physical Education School of the Federal University of Rio de Janeiro (EEFD/UFRJ). The inclusion criteria were: subjects aged over 60 years, literate, with a previous clinical diagnosis of either MDD or AD, and healthy elderly subjects with no previous history of psychiatric disease. Exclusion criteria included: depressive symptoms (in the healthy group); cognitive impairment (in the MDD group); previous cerebrovascular infarction; other types of dementia; important physical limitations by other pathologies or associated neurological diseases and severe visual and/or auditory disorders.

\section{Ethics}

All participants gave informed consent, which described all the research information and the contact of the researcher responsible for the study. This study was approved by the IPUB/ UFRJ Ethics Committee (no 45-Liv. 3-10) and it is part of a major study, entitled "The effect of aerobic exercise on salivary levels of cortisol and DHEA in older adults with Depression, Parkinson and Alzheimer". 


\section{Procedures and tests}

All assessments were performed during a single visit to the laboratory. Every experimental procedure was explained to the participants and their caregivers. After that, subjects answered an anamnesis, depressive symptoms rating scale, global cognitive status assessment and physical activity questionnaire. Subsequently, subjects performed the functional capacity test, which was applied by either a physical education professional or physiotherapist. The cognitive assessments were administered by a neuropsychologist.

\section{Anamnesis}

A well-structured instrument was applied, containing personal data such as name, age, family history, personal history and medications used by the subject.

\section{Hamilton Scale (HAM-D)}

The Portuguese version of the HAM-D ${ }^{17}$ was used. This scale is composed by 17 items, each one ranging from 0 to 4 points, based on symptom severity. Patients with scores higher than 25 were considered severely depressed; patients who obtained scores from 18 to 24 were considered moderately depressed; scores from 7 to 17 were classified as mildly clinical depressed and scores below 7 indicated remitted or no depression.

\section{Mini-Mental State Examination (MMSE)}

The MMSE consists of 11 simple questions or tasks grouped into 7 cognitive domains. Each domain assesses specific cognitive functions. Scores range from 0 to 30 and are used to provide an individual's present cognitive performance based on direct observation of the completion of test items/tasks. Cognitive performance is higher when higher MMSE values are reached ${ }^{18}$.

\section{Veterans Specific Activity Questionnaire (VSAQ)}

The Portuguese version of the Veterans Specific Activity Questionnaire (VSAQ) ${ }^{19}$ was applied to assess physical fitness without exercise. The VSAQ is a brief, easily understandable, questionnaire, consisting of a list of physical activities with scores ranging from 1 to 13 , according to their metabolic equivalents (METs). The respondent indicates which of the listed activities would cause fatigue or shortness of breath. The Nomogram, developed by Myers et al., which considers age and functional capacity based on the VSAQ, was also assessed $^{12}$. The regression equation used to calculate the nomogram is given by nomogram $=4.7+0.97$ (VSAQ, in METs) 0.06 (age). In this study, when there was divergence between the AD patient's answer and the caregiver's answer, it was considered the answer of the latter.

\section{International Physical Activity Questionnaire (IPAQ)}

In the present study, the short form (IPAQ-SF) was used, which assesses specific kinds of activities, such as walking, moderate-intensity activities and vigorous-intensity activities. All scores are expressed in MET-minutes/week. There are three possible classifications: insufficiently active (up to $600 \mathrm{METS} /$ week); sufficiently active (between 601 and 1500 METS/week); and very active (more than 1500 METS/week) ${ }^{20}$.

\section{2-minute Step test}

This test is included in the Senior Fitness Test, a simple battery of tests to assess the level of independence in Activities of Daily Living in Older Adults ${ }^{11}$. Used to assess aerobic endurance, the 2-minute step is an alternate test to the 6-minute walk test, and may be used when there are space limitations. A mark is drawn on a wall or door with the use of tape, respectively midway between the subject's patella and the iliac crest. After the signal "go", the participant must alternate steps, without moving forward, until the scheduled time. The score is the number of times the right knee reaches the required height in 2 minutes.

\section{Statistical analyses}

The normality and homoscedasticity of the sample were analyzed by the Shapiro-Wilk and Levene tests, respectively. A one-way ANOVA, for parametric data, and the KruskallWallis test, for non-parametric data, were used to conduct comparisons among the groups for each dependent variable (nomogram VSAQ and Step). To determine differences among the groups, a Bonferroni post hoc and Mann-Whitney test were applied. Multiple linear regression procedures were performed, adjusted for age and educational level and also for HAM-D and MMSE, to identify the influence of these variables on the relationships among the groups for both the nomogram VSAQ and Step test. The relationship between factors was analyzed using the Spearman correlation coefficient. The statistical analyses were conducted using the SPSS ${ }^{\circ}$ software package version 17.0 (IBM Corporation, New York, USA). The graphic illustrations of the analyses were prepared with Graph Pad Prism version 5.01 (Graph Pad Software, Inc., La Jolla, CA, USA). The significance level accepted in this study was $p \leq 0.05$.

\section{RESULTS}

The sample characteristics are shown in Table 1.

The subjects from the MDD and AD groups were older than the healthy subjects ( $p=0.014 ; p<0.001$, respectively), but non-significant differences between the MDD and AD groups were observed. The AD group showed less education years than the healthy group ( $p=0.022)$, but the difference was non-significant in comparison to the MDD group. The MDD group showed a higher number of associated comorbidities when compared to both the Healthy $(p=0.019)$ and AD $(p<0.001)$ groups. Moreover, the MDD group also 
Table 1. Descriptive analyses of the samples

\begin{tabular}{|c|c|c|c|c|c|}
\hline Groups & $\begin{array}{l}\text { Healthy } \\
(n=20)\end{array}$ & $\begin{array}{c}\text { MDD } \\
(n=20)\end{array}$ & $\begin{array}{c}\text { AD } \\
(n=17)\end{array}$ & $x^{2}$ & pvalue \\
\hline Age ${ }^{€}$ (years) & $64(60-81)$ & $74(60-85)$ & $78(68-89)$ & 17.868 & $<0.001^{*}$ \\
\hline Educational level ${ }^{\dagger}$ (years) & $13.5(4-23)$ & $11(4-16)$ & $8(1-15)$ & 6.315 & $0.043^{*}$ \\
\hline BMI $\left(\mathrm{kg} / \mathrm{m}^{2}\right)$ & $\begin{array}{c}24.83 \\
(22.31-34.16)\end{array}$ & $\begin{array}{c}24.83 \\
(20.33-35.75)\end{array}$ & $\begin{array}{c}25.14 \\
(19.76-26.64)\end{array}$ & 2.715 & 0.257 \\
\hline Diseases $(n)^{*}$ & $1(0-4)$ & $2(0-4)$ & $1(0-2)$ & 18.945 & $<0.001^{*}$ \\
\hline Medication $(n)^{\epsilon \varsigma}$ & $2(0-9)$ & $4.5(1-9)$ & $3(1-6)$ & 17.419 & $<0.001^{*}$ \\
\hline Women (\%) & 85.0 & 95.0 & 64.7 & & \\
\hline \multicolumn{6}{|l|}{ PA (level) } \\
\hline Insufficiently active (\%) & 20 & 45 & 41.2 & & \\
\hline Sufficiently active (\%) & 53.3 & 40 & 47.1 & & \\
\hline Very active (\%) & 26.7 & 15 & 11.8 & & \\
\hline HAM-D (score) ${ }^{* t}$ & $0.5(0-5)$ & $13(0-26)$ & $3(0-13)$ & 24.340 & $<0.001^{*}$ \\
\hline MMSE (score) $)^{f}$ & $28.5(23-30)$ & $29(22-30)$ & $21(12-28)$ & 26.592 & $<0.001^{*}$ \\
\hline IPAQ (METs/week) ${ }^{\dagger}$ & $1038(358-1680)$ & $735(200-1746)$ & $528(198-2178)$ & 6.03 & $0.049^{*}$ \\
\hline
\end{tabular}

Values expressed as median (minimum - maximum). X² = Chi - squared; BMI: body mass index. MDD: major depressive disorder; AD: Alzheimer disease; PA: physical activity; HAM-D: Hamilton Rate Scale: MMSE: Mini-Mental State Examination. ${ }^{*} p \leq 0.05=$ significant difference. $€$ : Significant difference between Healthy versus MDD and AD. t: Significant difference between Healthy and AD. $¥:$ Significant difference between MDD versus Healthy and AD. $\$:$ Significant difference between MDD and AD. $€$ : Significant difference between AD versus Healthy and MDD.

showed higher medication intake when compared to the healthy group $(p<0.001)$. All subjects of the MDD group were using antidepressants (19 used selective serotonin reuptake inhibitor - SSRI; 1 used serotonin-norepinephrine reuptake inhibitor - SNRIs).

Figure 1 shows the Step and nomogram VSAQ scores for each group. A significant difference among the groups was observed for the nomogram VSAQ (Healthy group = $7.91 \pm 1.87 ; \mathrm{MDD}=5.02 \pm 1.64 ; \mathrm{AD}=5.43 \pm 1.43 ; \mathrm{F}=17.256$, $\mathrm{p}<0.001$ - values in mean \pm standard deviation) and Step test (Healthy group $=60(35-82) ; \mathrm{MDD}=41(25-75) ; \mathrm{AD}=34$ $(21-69) ; X^{2}=10.153 ; p=0.006-$ values in median (minimum
- maximum)). The nomogram VSAQ and Step test showed significantly higher values in the healthy group, when compared to the MDD and AD groups, for both variables. Only non-significant differences between the MDD and AD groups were observed for all the tested variables. Considering the cut-off point of the Step test for risk for loss of functional mobility (65 repetitions), $82.4 \%$ of all subjects were included in this risk category (Healthy group $=80 \%$; $M D D=$ $85 \%$; $A D=82 \%)$. However, considering VSAQ answers, only $21 \%$ of all subjects were below 4 METs, considered as a cutoff point at risk of independence loss (Healthy group $=0$; $M D D=45 \% ; A D=17.6 \%)$.
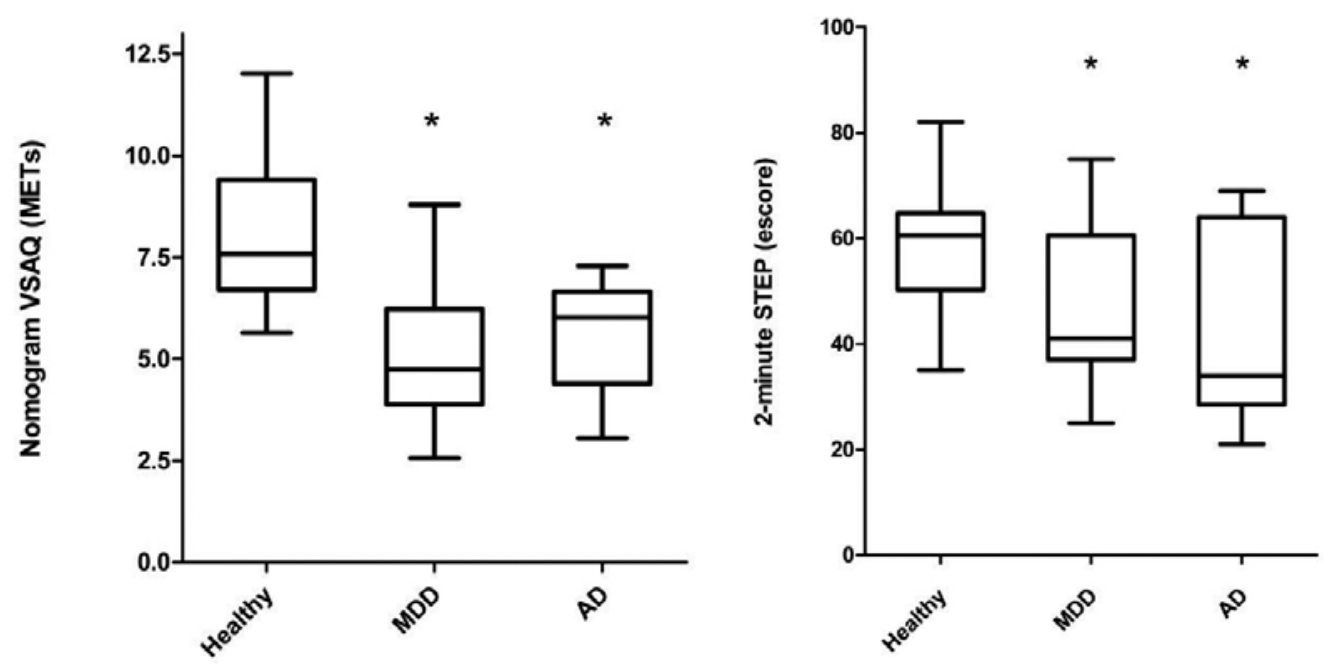

${ }^{*} p<0.05$. Significant difference compared to Healthy group. (Left) Comparisons of Nomogram VSAQ among groups. (Right) Comparisons of 2-minute Step Test among groups. MDD = major depressive disorder. $A D=$ Alzheimer disease.

Figure 1. Comparisons of Nomogram VSAQ and Step Test among groups 
Tables 2 and 3 show the results of the multiple linear regressions. The differences among groups remained for the nomogram VSAQ, even when adjusted for age and educational level or when adjusted for HAM-D and MMSE. However, the Step test showed non-significant differences among the groups after adjusting for age and educational level. The difference between the healthy and AD groups for the Step test did not remain when adjusted for MMSE.
The nomogram VSAQ showed a significant moderate correlation with the Step test considering all subjects. However, correlations observed for each group showed a significant relationship only in the AD group (Table 4). The healthy and MDD groups showed only non-significant correlations between the nomogram VSAQ and Step test.

Table 2. Linear regression for the nomogram VSAQ among groups

\begin{tabular}{|c|c|c|c|c|}
\hline Independent Variables & B coefficient & Confidence Interval (95\%) & $\mathrm{R}^{2}$ & $p$ value \\
\hline \multicolumn{5}{|l|}{ Diagnostic } \\
\hline Healthy & Reference & & 0.390 & \\
\hline MDD & -2.891 & $-3.950 /-1.832$ & & $<0.001$ \\
\hline$A D$ & -2.478 & $-3.583 /-1.374$ & & $<0.001$ \\
\hline \multicolumn{5}{|l|}{ Diagnostic* } \\
\hline Healthy & Reference & & 0.444 & \\
\hline MDD & -2.492 & $-3.612 /-1.372$ & & $<0.001$ \\
\hline$A D$ & -1.698 & $-3.048 /-0.347$ & & 0.015 \\
\hline \multicolumn{5}{|l|}{ Diagnostic ** } \\
\hline Healthy & Reference & & 0.398 & \\
\hline MDD & -2.888 & $-3.950 /-1.826$ & & $<0.001$ \\
\hline$A D$ & -2.038 & $-3.558 /-0.518$ & & 0.010 \\
\hline \multicolumn{5}{|l|}{ Diagnostic *** } \\
\hline Healthy & Reference & & 0.394 & \\
\hline MDD & -2.608 & $-4.046 /-1.169$ & & 0.001 \\
\hline$A D$ & -2.397 & $-3.543 /-1.250$ & & $<0.001$ \\
\hline
\end{tabular}

* Adjusted for age and educational level; ${ }^{* *}$ Adjusted for MMSE; *** Adjusted for HAM-D. MDD: major depressive disorder; AD: Alzheimer disease.

Table 3. Linear regression for the Step test among groups

\begin{tabular}{|c|c|c|c|c|}
\hline Independent Variables & B coefficient & Confidence Interval (95\%) & $\mathrm{R}^{2}$ & $\mathrm{p}$ value \\
\hline \multicolumn{5}{|l|}{ Diagnostic } \\
\hline Healthy & Reference & & & \\
\hline MDD & -12.300 & $-21.659 /-2.941$ & 0.208 & 0.011 \\
\hline$A D$ & -17.632 & $-27.395 /-7.870$ & & 0.001 \\
\hline \multicolumn{5}{|l|}{ Diagnostic* } \\
\hline Healthy & Reference & & & \\
\hline MDD & -8.267 & $-18.072 / 1.539$ & 0.291 & 0.097 \\
\hline$A D$ & -9.794 & $-21.616 / 2.028$ & & 0.102 \\
\hline \multicolumn{5}{|l|}{ Diagnostic ** } \\
\hline Healthy & Reference & & 0.283 & \\
\hline MDD & -12.225 & $-21.216 /-3.235$ & & 0.009 \\
\hline$A D$ & -7.279 & $-20.141 / 5.583$ & & 0.261 \\
\hline \multicolumn{5}{|l|}{ Diagnostic $* * *$} \\
\hline Healthy & Reference & & 0.208 & \\
\hline MDD & -13.025 & $-25.777 /-0.273$ & & 0.045 \\
\hline$A D$ & -17.842 & $-28.003 /-7.680$ & & 0.001 \\
\hline
\end{tabular}

*Adjusted for age and educational level; ** Adjusted for MMSE; *** Adjusted for HAM-D. MDD: major depressive disorder; AD: Alzheimer disease. 
Table 4. Spearman's correlation between the VSAQ nomogram and Step test

\begin{tabular}{cccccc}
\hline & \multicolumn{3}{c}{ Step } \\
\cline { 2 - 5 } Nomogram VSAQ & All $(n=57)$ & Healthy $(n=20)$ & MDD $(n=20)$ & AD $(n=17)$ \\
\cline { 2 - 5 } & $0.448(p<0.001)^{*}$ & $0.121(0.613)$ & $0.151(0.526)$ & $0.608(0.01)^{*}$ \\
\hline
\end{tabular}

Rs values ( $p$ values); ${ }^{*} p \leq 0.05$. MDD: major depressive disorder; AD: Alzheimer disease.

\section{DISCUSSION}

In the present study, our objective was to assess cardiorespiratory capacity through both subjective and objective tests among older adults diagnosed with either MDD or AD and healthy older adults. Moreover, we aimed to analyze the influence of some confounder variables on the differences among the groups. Despite the lower cardiorespiratory fithess of elderly patients with MDD and AD found in both evaluations, these tests showed low correlation and very different risk classifications of functional loss. In addition, age, educational level and cognitive performance are variables that can influence the performance objective evaluation. The inferior cardiorespiratory capacity in MDD older adults observed in the present study corroborates previous findings, which have shown that MDD is associated with impaired physical skill5. Regarding the AD group, our data also agree with literature, showing an inferior performance in this group when compared to healthy older adults. A previous study found that AD patients had lower peak cardiorespiratory responses $\left(\mathrm{VO}_{2 \text { peak }}\right)$ compared to individuals without dementia ${ }^{21}$. However, the fact that the healthy older adults analyzed in the present study are younger than those in the other two groups must be taken into account, since this difference might influence the results. Extensive discussions in the literature regarding the normal physiological decline in cardiorespiratory capacity during the aging process are available, and data indicate that this physical skill decreases by approximately 9\%-10\% per decade ${ }^{7}$. Although this decline is not constant across the age span, with each successive age decade this decline has been shown to accelerate ${ }^{22,23}$.

Contrary to our expectations, the differences among groups for the nomogram VSAQ were not influenced by depressive symptoms or cognitive decline. Cognitive impairment, present in the AD group, was expected to cause an overestimated response in this subjective assessment, since this brain disorder is characterized by memory decline, disorientation, confusion and planning disorders ${ }^{24}$. However, it is important to note these patients were helped by caregivers to answer this questionnaire, which can be minimized the influence of the cognitive impairment of AD patients. In this way, the cognitive decline observed in AD patients only influenced the performance of objective tests. Although the main objective of the Step test is to assess aerobic capac- ity, cognitive impairment may influence the results, since progressive cognition declines may cause a disorganization of the brain network that controls locomotion, leading to impaired gait time ${ }^{25}$. Corroborating these findings, another study found declines in the motor function of patients with different $A D$ stages $^{6}$.

Underestimated results for the nomogram VSAQ were expected for the MDD group, since this mood disorder is characterized by altered self-esteem, low energy and self-depreciative tendencies, common characteristics in MDD patients ${ }^{26}$. However, the early hypothesis of the present study was not confirmed, since depressive symptoms did not influence the subjective assessments. Therefore, it seems that the nomogram VSAQ may be the method that presents less influence of variables such as global cognitive status and depressive symptoms.

The data obtained in the present study demonstrated significant correlations between the subjective and objective measurements of cardiorespiratory capacity, considering all individuals. This corroborates a previous research conducted in our laboratory with healthy older adult women ${ }^{16}$, where significant correlations between the VSAQ and the 6-minute walk test, a similar test to assess aerobic resistance in elderly people, were also reported ${ }^{9,15}$. Contrary to our hypothesis, the healthy group showed non-significant correlations between the nomogram VSAQ and the Step test. This group presented very low Step test performances, based on cut-off points for the maintenance of physical independence in older adults ${ }^{27}$. Therefore, the healthy group may be classified as being at risk of functional dependence, suggesting very low cardiorespiratory capacity. However, the mean score of the nomogram VSAQ in the present study was close to $8 \mathrm{METs}\left(\mathrm{VO}_{2 \max }=28 \mathrm{ml} \cdot \mathrm{kg}^{-1} \cdot \mathrm{min}^{-1}\right)$, higher than the minimum of approximately 4-5 METs $\left(\mathrm{VO}_{2 \max }=15-18 \mathrm{ml} \cdot \mathrm{kg}^{-1} \cdot \mathrm{min}^{-1}\right)$ needed for maintenance of independence in activities of daily living in elderly subjects ${ }^{22}$. Thus, we believe that the nomogram VSAQ was overestimated by the healthy group, which seems to be in agreement with a previous study that pointed out lower VSAQ accuracy in individuals with cardiorespiratory capacity lower than $4 \mathrm{METS}^{13}$. This may also explain the absence of significant correlations between the variables in the MDD group. Only the AD group showed significant correlations, contrary to our early hypothesis. Subjects with higher cognitive impairment presented lower scores in both 
measurements, while those with lower cognitive disorders tended to show better functional performance. This may explain the higher score variability in the AD group and, thus, the correlations between the tests. However, it is important to note that the AD patients were influenced by their caregivers to answer the $V S A Q$, which aided in choosing the more realistic option of cardiorespiratory capacity and, consequently, might have influenced the score accuracy in this group.

To the best of our knowledge, this is the first study to use both objective and subjective cardiorespiratory measurement tools in older adults diagnosed with MDD and/or AD, providing comparisons with healthy older adults. These tools are alternatives to the considered gold-standard method used to assess cardiorespiratory fitness (the ergospirometry test) also in clinical populations. The main objectives were to lower costs and to facilitate the application of this measurement. For example, a submaximal protocol to assess cardiorespiratory fitness in older adults with mood disorders has been demonstrated as being a satisfactory validity criterion and of simple implementation when compared to ergospirometry ${ }^{28}$. However, even this submaximal protocol demands a physician and specific equipment, such as a treadmill, for its execution. Specifically for people with dementia, this measurement may be harder to perform, since they show cognitive status impairment, which is not observed in healthy older adults and those with mood disorders. This study presented an easier objective assessment tool to measure the aerobic capacity in these patient groups. In this sense, the 6-minute walk test has been considered sensitive enough to measure the endurance capacity of older patients with dementia ${ }^{29}$. However, this test demands a 30 meter-length walking course, making it difficult to use in some facilities, clinics and hospitals. In this sense, the Step test has been shown as being a good alternative to assess the same physical capacity, besides showing good correlation with the 6-minute walk test in older adults ${ }^{11}$. However, considering the weak correlations between the tests used in this study, it is necessary caution with these tools to clinical application, especially in healthy elderly and depressive subjects. Moreover, the majority of subjects presented at risk of functional loss, measured through the objective test, were ranked above the independence level, when measured by subjective evaluation. In addition, age, educational level and MMSE scores can influence the performance of the Step test. Using the gold standard for assessing cardiorespiratory capacity (directly measure maximal oxygen intake) remains as a more accurate option.

This study presented some limitations that should be taken into account, such as: the fact that the cross-sectional design is unable to provide a cause-effect relationship between variables; there was no direct measurement of cardiorespiratory capacity $\left(\mathrm{VO}_{2 \max }\right)$, recognized as the gold standard assessment for this physical skill. Moreover, even though the sample size is in agreement with previous studies, the number of subjects in each group may have influenced the results. In addition, our sample had only few male subjects per group which limits the extrapolation of the results for older men.

\section{CONCLUSIONS}

Although the lower cardiorespiratory fitness of older adults with MDD and AD could be observed through subjective and objective evaluations, the results should be viewed with caution, since the tests showed low correlation and different risk classifications of functional loss. Moreover, it is important to note that age, education level and cognitive ability are variables that can influence the performance of the objective evaluation.

\section{INDIVIDUAL CONTRIBUTIONS}

Marcos Felipe Zanco - Contributed to conception and design of the study and analysis and interpretation of the data; had substantially contributed to drafting the article; and had given the final approval of the version to be published.

Helena Moraes - Contributed to conception and design of the study; revised it critically for important intellectual content; and had given the final approval of the version to be published.

Geraldo Maranhão Neto - Contributed to analysis and interpretation of data; revised it critically for important intellectual content; and had given the final approval of the version to be published.

Jerson Laks - Contributed to analysis and interpretation of data; revised it critically for important intellectual content; and had given the final approval of the version to be published.

Andrea Camaz Deslandes - Contributed to conception and design of the study; revised it critically for important intellectual content; and had given the final approval of the version to be published.

\section{CONFLICTS OF INTEREST}

The authors have no conflicts of interest to report.

\section{ACKNOWLEDGMENTS}

This study was supported by grants received from Fundação de Amparo à Pesquisa do Rio de Janeiro (FAPERJ) and Conselho Nacional de Desenvolvimento Científico e Tecnológico (CNPq) (E-26/100.274/2013, E-26/102.174/2013, E-26/112.631/2012). 


\section{REFERENCES}

1. Djernes JK. Prevalence and predictors of depression in populations of elderly: a review. Acta Psychiatr Scand. 2006;113(5):372-87.

2. Blay SL, Andreoli SB, Fillenbaum GG, Gastal FL. Depression morbidity in later life: prevalence and correlates in a developing country. Am J Geriatr Psychiatry. 2007;15(9):790-9.

3. Herrera E, Jr Caramelli P, Silveira ASB, Nitrini R. Epidemiologic survey of dementia in a community-dwelling Brazilian population. Alzheimer Dis Assoc Disord. 2002;16(2):103-8.

4. Penninx BW, Guralnik JM, Ferrucci L, Simonsick EM, Deeg DJ, Wallace RB. Depressive symptoms and physical decline in community-dwelling older persons. JAMA. 1998;279(21):1720-6.

5. Hollenberg M, Haight T, Tager IB. Depression decreases cardiorespiratory fitness in older women. J Clin Epidemiol. 2003;56(11):1111-7.

6. Zidan M, Arcoverde C, Araújo NB, Vasques P, Rios A, Laks J, et al. Motor and functional changes in different stages of Alzheimer's disease. Rev Psiquiatr Clín. 2012;39(5):161-5.

7. Chodzko-Zajko WJ, Proctor DN, Fiatarone Singh MA, Minson CT, Nigg CR, Skinner JS. American College of Sports Medicine position stand. Exercise and physical activity for older adults. Med Sci Sports Exerc. 2009;41(7):1510-30.

8. Maranhão-Neto GA, Ponce de Leon ACM, Farinatti PTV. Cross-cultural equivalence of three scales used to estimate cardiorespiratory fitness in the elderly. Cad Saúde Pública. 2008;24(11):2499-510.

9. Myers J, Zaheer N, Quaglietti S, Madhavan R, Froelicher V, Heidenreich P. Association of functional and health status measures in heart failure. J Card Fail. 2006;12(6):439-45.

10. Myers J, Bader D, Madhavan R, Froelicher V. Validation of a specific activity questionnaire to estimate exercise tolerance in patients referred for exercise testing. Am Heart J. 2001;142(6):1041-6.

11. Rikli RE, Jones CJ. Development and validation of a functional fitness test for communityresiding older adults. J Aging Phys Act. 1999;7:129-61.

12. Myers J, Do D, Herbert W, RibisI P, Froelicher VF. A nomogram to predict exercise capacity from a specific activity questionnaire and clinical data. Am J Cardiol. 1994;73(8):591-6.

13. Bader DS, McInnis KJ, Maguire TE, Pierce GL, Balady GJ. Accuracy of a pretest questionnaire in exercise test protocol selection. Am J Cardiol. 2000;85(6):767-70, A8-9.

14. McAuley P, Myers J, Abella J, Froelicher V. Evaluation of a specific activity questionnaire to predict mortality in men referred for exercise testing. Am Heart J. 2006;151(4):890.e1-7.

15. Kojima S, Wang DH, Tokumori K, Sakano N, Yamasaki Y, Takemura Y, et al. Practicality of Veterans Specific Activity Questionnaire in evaluation of exercise capacity of communitydwelling Japanese elderly. Environ Health Prev Med. 2006;11(6):313-20.
16. De Oliveira Brito LV, Maranhao Neto GA, Moraes H, Emerick RFES, Deslandes AC. Relationship between level of independence in activities of daily living and estimated cardiovascular capacity in elderly women. Arch Gerontol Geriatr. 2014;59(2):367-71.

17. Moreno RA, Moreno DH. Escalas de depressão de Montgomery \& Asberg (MADRS) e de Hamilton (HAM-D). Rev Psiquiatr Clín. 1998;25(5):262-72.

18. Brucki S, Nitrini R, Caramelli P, Bertolucci P, Okamoto, I. Sugestões para o uso do MiniExame do Estado Mental no Brasil. Arq Neuropsiquiatr. 2003;61(3-B):777-81.

19. Maranhão-Neto GA, Leon ACP, Farinatti PTV. Validity and equivalence of the Portuguese version of the Veterans Specific Activity Questionnaire. Arq Bras Cardiol. 2011; $97(2): 130-5$.

20. Benedetti T, Antunes PC, Rodriguez-Añes (R, Mazo GZ, Petroski EL. Reprodutibilidade e validade do Questionário Internacional de Atividade Física (IPAQ) em homens idosos. Rev Bras Med Esporte. 2007;13(1):11-6.

21. Billinger SA, Vidoni ED, Honea RA, Burns JM. Cardiorespiratory response to exercise testing in individuals with Alzheimer's disease. Arch Phys Med Rehabil. 2011; 92(12):2000-5.

22. Shephard RJ. Maximal oxygen intake and independence in old age. Br J Sports Med. 2009;43(5):342-6.

23. Fleg JL, Morrell CH, Bos AG, Brant LJ, Talbot LA, Wright JG, et al. Accelerated longitudinal decline of aerobic capacity in healthy older adults. Circulation. 2005;112(5):674-82.

24. Caramelli P, Barbosa MT. How to diagnose the four most frequent causes of dementia? Rev Bras Psiquiatr. 2002;24:7-10.

25. Sheridan PL, Hausdorff JM. The role of higher-level cognitive function in gait: executive dysfunction contributes to fall risk in Alzheimer's disease. Dement Geriatr Cogn Disord. 2007;24(2):125-37.

26. Stella F, Gobbi S, Corazza DI, Costa JLR. Depressão no idoso: diagnóstico, tratamento e benefícios da atividade física. Motriz. 2002;8(3):91-8.

27. Rikli RE, Jones CJ. Development and validation of criterion-referenced clinically relevant fitness standards for maintaining physical independence in later years. Gerontologist. 2013;53(2):255-67.

28. Oliveira NA, Silveira HS, Carvalho A, Hellmuth CGS, Santos TM, Martins JV, et. al. Assessment of cardiorespiratory fitness using submaximal protocol in older adults with mood disorder and Parkinson's disease. Rev Psiquiatr Clín. 2013;40(3):88-92.

29. Bossers WJR, van der Woude LHV, Boersma F, Scherder EJA, van Heuvelen MJG. Recommended measures for the assessment of cognitive and physical performance in older patients with dementia: a systematic review. Dement Geriatr Cogn Dis Extra. 2012;2(1):589609. 